ÉGYPTE

monde arabe

\section{Égypte/Monde arabe}

4 | 2007

Figures de la santé en Égypte

\title{
Sur la réédition de la Description de l'Égypte en DVD
}

Note critique

\section{Nicolas de Lavergne}

\section{(2) OpenEdition}

Journals

Édition électronique

URL : https://journals.openedition.org/ema/1788

DOI : $10.4000 /$ ema. 1788

ISSN : 2090-7273

Éditeur

CEDEJ - Centre d'études et de documentation économiques juridiques et sociales

Édition imprimée

Date de publication : 31 décembre 2007

Pagination : 345-352

ISBN : 2-6905838-43-4

ISSN : 1110-5097

Référence électronique

Nicolas de Lavergne, «Sur la réédition de la Description de l'Égypte en DVD », Égypte/Monde arabe [En ligne], 4 | 2007, mis en ligne le 08 juillet 2008, consulté le 07 juillet 2022. URL : http://

journals.openedition.org/ema/1788; DOI : https://doi.org/10.4000/ema.1788 


\title{
SUR LA RÉÉDITION DE LA DESCRIPTION DE L'ÉGYPTE EN DVD
}

\author{
NOTE CRITIQUE
}

À propos de la réédition en DVD de la Description de l'Égypte, publiée par les ordres de sa Majesté l'Empereur Napoléon le Grand, édition impériale 1809-1828, version numérique intégrale, interrogeable en plein texte. Premier volume de la collection "Les grandes expéditions scientifiques du 19e siècle ", dirigée par Jean-Yves Empereur, éditions Harpocrate ${ }^{1,} 2006,1$ DVD, $35 €$.

Ce DVD présente, sous forme de documents PDF, la reproduction des vingt volumes de la première édition de la Description de l'Égypte, soit 9500 pages de texte et un millier de planches environ. Le texte de la Description n'avait jamais été réédité depuis la seconde édition, en plus petit format que la première, dans les années 1810-1830. Cette réédition est donc d'ores et déjà un événement en ce qui concerne le texte.

La réédition sur papier de la Description de l'Égypte est un projet qui remonte à plus de trente ans et n'a jamais vu le jour sous une forme complète. II faut savoir que la monumentalité des deux premières éditions, publiées presque simultanément durant deux décennies, dans deux formats différents, juste après l'expédition elle-même, sous la direction de EdmeFrançois Jomard - lui-même ancien membre de l'expédition et éminent représentant de l'égyptomanie française jusque dans les années 1860 - a de facto empêché toute réédition complète ultérieure. La seconde édition, par le libraire Panckoucke, dans un format réduit déjà plus accessible (elle est dite « portative ») mais qui restait encombrant, n'a été tirée qu'à 3000 exemplaires. Depuis près de deux siècles, la Description n'était donc accessible que dans ces deux éditions volumineuses et fragiles publiées sous l'Empire et la Restauration. Un projet de réédition intégrale a bien vu le jour en 1975,

1. http://www.harpocrate.eu.com. 
à la suite du voyage du Président français Valéry Giscard d'Estaing chez son homologue Anouar al-Sadate, mais il a été finalement été abandonné pour des raisons financières ${ }^{2}$. Aucune réédition sur papier des 9500 pages de texte n'avait donc jusqu'ici paru.

Quant aux planches, elles ont connu plusieurs éditions plus ou moins complètes et pratiques depuis trois décennies. On peut compléter Jean-Pierre Corteggiani, qui en signale deux, parues en 1988 (Hazan et Institut d'Orient), par John von B. Rodenbeck, qui en cite cinq, entre 1975 et $1994^{3}$ :

- Angèle Kapoîan-Kouymjian et Dikran Kouymjian, The splendor of Egypt: A commentary on Napoléon Bonaparte's Expedition (17981801) and the Description de l'Égypte, Delmar, N. Y., Caravan Books, 1975 (100 reproductions dans un luxueux coffret).

- Robert Anderson et Ibrahim Fawzi (dirs), Egypt in 1800: Scenes from Napoleon's Description de l'Égypte, Londres, à compte d'auteur puis Barrie \& Jenkins, AUC [as Egypt revealed: Scenes from Napoleon's Description de l'Égypte], 1987 (174 illustrations).

- Charles Gillispie et M. Dewachter (dirs), Monuments of Egypt: Description de l'Égypte: The Napoleonic Edition, Princeton, Princeton architectural Press, 1987 ; édition française, Monuments de l'Égypte, l'édition impériale de 1809, Paris, Hazan, 1988 (uniquement reproduction des planches de l'Antiquité).

- Michel Sidhom (dir.), Description de l'Égypte, volume I [sic], Paris, Institut d'Orient, 1988 (reproduction de l'ensemble des planches, en un seul épais volume difficile à consulter)

- Gilles Néret (dir.), Description de l'Égypte, publiée par les ordres de Napoléon Bonaparte, traduction anglaise par Chris Miller, allemande par Bettina Blumenberg, Cologne, Benedikt Taschen Verlag $\mathrm{GmbH}$, 1994 (édition en 14x19,5 qui reproduit toutes les planches en format réduit; c'est l'édition française que l'on trouve aujourd'hui le plus couramment).

Comme le souligne J.-P. Corteggiani, aucune de ces éditions ne reprend l'explication des planches qui les accompagnaient dans l'édition originale ${ }^{4}$. Les notes explicatives sont souvent très réduites, voire pour la dernière édition (Taschen) complètement absentes. Pour consulter les planches, il fallait choisir entre l'original énorme et poussiéreux et une sélection de planches en petit format presque sans commentaire. Quant au texte, il n'y avait que l'édition originale.

2. J.-P. Corteggiani, «Description de l'Égypte », Lettre d'information oucc, n 18 , septembre 1989, p. 4.

3. John von B. Rodenbeck, «An orientalist monument reconsidered » dans Jason Thompson (dir.), Egyptian encounters, Le Caire, AUC, "Cairo Papers in Social Sciences ", volume 23, n 3, 2002, p. 91.

4. J.-P. Corteggiani, op. cit., p. 4. 
Il a fallu attendre les progrès de l'édition électronique pour que soit lancée au Centre d'études alexandrines $\left(\mathrm{CEA}^{5}\right)$, à Alexandrie, à partir de 1996, la numérisation de l'ensemble de la Description de l'Égypte. L'entreprise, sous la direction de Jean-Yves Empereur, a duré dix ans. Qu'en est-il ? Cette édition est-elle utile? Pourquoi rééditer aujourd'hui la Description de l'Égypte?

Si l'on s'entend sur ce qu'est la Description, alors cette réédition est utile. Ce n'est certes pas la première description de l'Égypte, comme le soulignent par exemple Maurice Martin ${ }^{6}$ et John von B. Rodenbeck ${ }^{7}$, mais quoi qu'en dise ce dernier, en 9500 pages, sur l'état antique, moderne et naturel, c'est la plus étendue ${ }^{8}$. Ce n'est pas non plus la dernière ; Rodenbeck insiste à juste titre sur les quelques trois cents voyageurs qui ont publié des relations de voyage en l'Égypte ou l'incluant, des années 1810 aux années $1860^{9}$ - qu'ils visitent le Proche-Orient ou prennent la route (devenue plus sûre) de I'Inde. Et surtout, elle a sans doute été moins séminale que ne l'aurait voulu, par exemple, un Edward Saïd, justement critiqué par Rodenbeck pour ses à-peuprès hâtifs (Chateaubriand inspiré par la Description, alors qu'il a effectué son voyage en Égypte trois ans avant la parution du premier volume...). Toujours selon Rodenbeck, de nombreuses reproductions de hiéroglyphes sont erronées, ayant été réalisées par des artistes avant leur décryptage ${ }^{10}$; de nombreux détails de l'état antique et de l'état moderne sont soit caducs lors de la parution de la Description, vingt ou trente ans plus tard, soit le fruit de l'imagination des auteurs ou des artistes. Cependant, in fine et non sans humour, Rodenbeck reconnaît certains mérites à la Description :

For the historically or even just the nostalgically minded, the Description is full of marvels. Its best articles offer a plenitude of information much in the style of the Encyclopédie, sympathetically gathered and sometimes

5. Voir les activités du CEA sur son site : http://www.cealex.org.

6. Maurice Martin, "Aux débuts de la description moderne de l'Égypte », D'un Orient l'autre, vol. I - Configurations, Paris, Éditions du CNRS, 1991, p. 343-350. Une sélection d'articles, comprenant celui-ci, de Maurice Martin est en cours d'édition au Cedej.

7. John von B. Rodenbeck, op. cit., p. 90-132.

8. Signalons l'entreprise scientifique, une cinquantaine d'années plus tôt, de Forskäl, Niebuhr et $c^{\text {ie }}$ qui ont passé plusieurs mois en Égypte avant de s'embarquer pour l'Arabie dont seul l'arpenteur Carl Niebuhr est finalement revenu, expédition dont les résultats n'ont jamais été publiés, mais qui auraient constitué, en tout cas en ce qui concerne l'histoire naturelle, un important précédent à la quatrième partie de notre Description de l'Égypte. Un beau récit a été tiré par Hansen Thorkild des archives de cette expédition : La mort en Arabie : une expédition danoise, 1761-1766, Arles, Actes Sud, 1993.

9. John von B. Rodenbeck, op. cit., p. 126-129.

10. John von B. Rodenbeck, op. cit., p. 117. 
available nowhere else. But the bulk of this great work, almost since its publication, has been not unlike many other Egyptian monuments: admirable, no doubt, and beautiful as an example of book production, but also inconveniently large, expensive to keep, and, after all, on a daily basis not overwhelmingly useful ${ }^{11}$.

Au gré de nombreux mémoires et descriptions rédigés lors de leur présence en Égypte ou après coup par les savants de l'expédition, certains présentés lors des séances de l'Institut d'Égypte et tous discutés lors des séances de la Commission impériale chargée de l'édition scientifique de l'ouvrage, de très nombreux sujets sont abordés. Les auteurs étaient des savants de toutes disciplines, certains déjà orientalistes, quand d'autres vont le devenir grâce à ce séjour.

La présente édition de la Description de l'Égypte comprend d'abord, concernant l' "Antiquité », deux tomes de descriptions, deux tomes de mémoires spécifiques et cinq tomes de planches. Les descriptions couvrent la plupart des sites, d'Assouan à la Méditerranée. De nombreux monuments ont depuis lors été désensablés, restaurés, ou détruits. Cette partie constitue donc aussi un état des lieux en 1800 des monuments de l'Antiquité pharaonique, hellénistique et romaine, avant l'intervention des égyptologues, des restaurateurs, des marchands et des voleurs,... Les mémoires abordent des questions très variées : instruments de musique, nilomètre, systèmes de mesure, zodiaque et astronomie, embaumement, inscriptions, pyramides, hiéroglyphes... Enfin, les planches présentent des vues panoramiques, des élévations, des détails architecturaux ou décoratifs.

Les cinq tomes relatifs à I' « état moderne » de l'Égypte ont un intérêt historique : ils présentent l'Égypte de 1800, vue par des savants français héritiers des Lumières et de l'encyclopédisme. Les 47 mémoires, notices, essais et tableaux sont souvent très détaillés ; ils traitent de nombreux sujets : mer Rouge et canal, ville de Qusayr, éclosion des poulets, médicaments et maladies endémiques, impôts et administration, finances et monnaies, inscriptions soufies, observations sur diverses régions ou tribus, musique et instruments, hydrographie, chronologies, poids et mesures, topographie, agriculture et commerce, cartographie, Alexandrie et le Caire... Je retiens deux mémoires en particulier : I' « Essai sur les mœurs des habitans modernes de l'Égypte », par Gilbert de Chabrol et la "Description abrégée de la ville et de la citadelle du Kaire, suivie de l'explication du plan de cette ville et de ses environs, et contenant des renseignements sur sa distribution, ses monumens, sa population, son commerce et son industrie », par l'inépuisable François-Edme Jomard, auteur par ailleurs de cartes, de nombreuses descriptions dans l'état antique et de plusieurs autres mémoires sur l'Égypte

11. John von B. Rodenbeck, op. cit., p. 131. 
moderne ${ }^{12} \ldots L^{\prime}$ « essai sur les mœurs » de Chabrol n'a pas connu la fortune du célèbre ouvrage publié une dizaine d'années plus tard en GrandeBretagne par Edward Lane: An Account on the Manners and customs of the Modern Egyptians $(1836)^{13}$, réédité cinq fois au long du xIx siècle et davantage encore au $x^{e}$ siècle, infiniment plus cité dans la littérature sur l'Égypte que son pendant français. Ce dernier n'était accessible, jusqu'à la présente édition électronique, que dans les deux éditions originales des années $1820 \ldots$

105 planches d'illustrations complètent cet " état moderne », suivies de 88 planches sur les arts et métiers, costumes et portraits, vases, meubles et instruments, inscriptions, monnaies et médailles, d'une façon très proche de celle de l'Encyclopédie de Diderot et d'Alembert.

On pourrait aussi tirer des volumes de l'état moderne, en rapport avec le thème du présent numéro d'Égypte/Monde arabe, les textes suivants : " Notice sur les médicamens usuels des Égyptiens », par M. Rouyer ; "Mémoires et Observations sur plusieurs maladies qui ont affecté les troupes de l'armée française pendant l'expédition d'Égypte et de Syrie, et qui sont endémiques dans ces deux contrées ${ }^{14} »$, par le baron Larrey, premier chirurgien de la Garde de l'Empereur; " Notice sur la conformation physique des Égyptiens et des différentes races qui habitent en Égypte, suivie de quelques réflexions sur l'embaumement des momies », par le même ; par ailleurs, le mémoire de Chabrol contient les chapitres " Des maladies principales », "Fécondité des femmes »,

12. Yves Laissus recense trente contributions de la main de Jomard, représentant 1400 pages, soit le tiers des textes publiés, ainsi que 8 cartes en collaboration et les dessins préparatoires qui remplissent, en tout ou en partie, 119 planches. Voir en particulier, sur cet homme peu connu mais prolifique et central dans l'égyptomanie française du XIX ${ }^{\mathrm{e}}$ siècle, I'abondante biographie de Y. Laissus : Jomard, le dernier Égyptien, Paris, Fayard, 2004, 654 p., avec chronologie, bibliographie, liste des œuvres de Jomard, index. Une somme qui en outre donne une bibliographie très complète des travaux et articles consacrés à la Description de l'Égypte.

13. Récemment réédité par I'Université américaine du Caire (Le Caire, AUC Press, 2003, 619 p.), qui a parallèlement publié sa Description of Egypt (Le Caire, AUC Press, 2000, 786 p.), qui couvre l'Égypte pharaonique et la vie contemporaine comme la Description napoléonienne, mais avait été refusée par les éditeurs londoniens à la fin des années 1820 (ce qui l'avait contraint à abandonner ce projet et à revenir au Caire rassembler les matériaux de Manners and Customs avant de poursuivre une carrière de lexicographe, d'éditeur et de traducteur de textes arabes).

14. Les maladies traitées sont les suivantes: ophtalmie endémique, tétanos traumatique, peste, hépatitis, atrophie des testicules, sarcocèle, fièvre jaune, lèpre et éléphantiasis, scorbut, syphilis. Larrey réfléchit aussi sur l'influence du climat égyptien sur les plaies, sur l'établissement d'un hôpital civil au Caire, sur la chirurgie et la médecine des Égyptiens, sur l'influence des saisons de l'Égypte sur la santé des individus. 
"Circoncision », "Bains publics »; le mémoire de Jomard, suscité, comprend un paragraphe sur les hôpitaux et un autre intitulé « De la population du Kaire, de la santé des habitans, et de la mortalité ».

L'Atlas qui suit comporte deux cartes d'Égypte, avec le tableau d'assemblage: une carte géographique en 3 feuilles et une autre, topographique, en 47 feuilles. Ce ne sont pas les premières cartes de l'Égypte, certes, mais leur précision et leur extension à une bonne partie de l'Égypte, grâce à l'occupation militaire et à la présence de plusieurs polytechniciens spécialistes d'arpentage et de trigonométrie, font que ces cartes constituent une référence pour toute étude historique ou géographique de l'Égypte moderne. En outre, la maniabilité de la carte numérisée, dans laquelle on peut circuler de manière aisée et opérer un zoom de bonne qualité jusqu'à $200 \%$, fait tout l'intérêt de cette édition en $D_{V D}{ }^{15}$. Les originaux étaient effectivement reproduits dans des volumes mesurant 75 par $108 \mathrm{~cm}$ dans la première édition !

La quatrième partie de la Description, intitulée "Histoire naturelle », comprend cinq volumes : deux contenant des mémoires et trois contenant les planches. Dans le premier tome, les mémoires traitent des sujets suivants: poissons du Nil, palmier Doum, oiseaux, reptiles, poissons de la mer Rouge et de la Méditerranée ; Savigny y publie ensuite deux tableaux systématiques sur les ascidies et sur les annélides. Le second tome comprend des mémoires sur les plantes spontanées et cultivées, les roches, les mammifères, la flore d'Égypte, les observations météorologiques, le limon du Nil ; il est composé enfin d'un essai conséquent de plus de 300 pages sur la «constitution physique de l'Égypte» (géographie, sol, minéraux, déserts, silice, natron...). Enfin, les trois volumes de planches présentent les mammifères, les oiseaux (14 belles planches en couleur), les reptiles, les poissons du Nil, puis les mollusques, les annélides, les crustacés, les arachnides, les insectes, les échinodermes, les zoophites, les ascidies, les polypes et les algues; le troisième volume de planches comprends la botanique (62 planches) et la minéralogie (15 planches en couleur), suivies d'explications détaillées sur ces planches. Toutes les planches de l'histoire naturelle ont été réalisées, à l'époque, à partir de magnifiques dessins fournis par Étienne Geoffroy Saint-Hilaire, Jules-César Savigny, Alire Raffeneau-Delile et HenriJoseph Redouté. Les gravures, en noir ou en couleur, sont toutes rendues avec une haute résolution, double de l'original, qui permet d'explorer les détails de ces planches.

Le DVD comprend en outre plusieurs outils fort utiles. Tout d'abord, une table générales des planches, agrémentée des légendes de chacune d'elles, à partir de laquelle un simple clic fait accéder à la planche choisie. Les cartes et les planches annexées aux volumes de texte y sont également recensées

15. On peut zoomer beaucoup plus avant sur les cartes et les illustrations, mais audelà de $200 \%$, l'image est de plus en plus imprécise et pixellisée. 
et accessibles. La navigation de volume en volume se fait de manière aisée grâce à Adobe Reader 7.0, un logiciel gratuit dont les fichiers d'installation sont présents sur le DVD. L'ensemble des documents, planches et textes, peut être copié sur le disque dur de l'ordinateur pour consultation ultérieure sans usage du DVD ${ }^{16}$.

Une interface permet en outre de faire des recherches en plein texte, d'une part dans les légendes des planches, d'autre part dans l'ensemble des textes. II va de soi qu'une telle fonction est très utile. L'apport de l'électronique se marque ici par rapport à une consultation sur le support papier. II y a cependant une limite technique à la recherche dans les textes, ce qui n'est pas le cas pour les légendes des planches. En effet, celles-ci ont été entièrement corrigées après le scannage ; cette opération était nécessaire afin de présenter un index des planches parfaitement clair.

Cependant, on peut critiquer la manière dont le texte est indexé, sur un plan technique. L'ensemble du texte a été scanné, et c'est le document scanné, au format pdf, qui est présenté - comme par exemple pour les textes présentés sur le site de la BNF (gallica.bnf. $\mathrm{fr}^{17}$ ). Cela permet effectivement de présenter un texte dans sa pagination originale et donc de le citer de manière rigoureuse dans l'édition originale. Comme les documents de Gallica, le texte a été indexé, et c'est sur cet index que la recherche est possible. Cependant, cet index, construit à partir d'un logiciel de reconnaissance automatique des caractères, n'a pas été corrigé et lissé, ce qui fait que de nombreux mots sont mal orthographiés, voire complètement erronés. On peut donc lire en texte intégral les 9500 pages (en pdf), mais on ne peut pas être sûr qu'une recherche opérée sur l'index du texte comprenne la totalité des occurrences du mot effectivement égaillées dans les volumes.

Un avantage considérable de cette réédition numérique est de mettre à la disposition du lecteur l'ensemble des textes publiés à l'époque dans la première édition de la Description de l'Égypte, mais sans aucune introduction historique ou présentation scientifique (contrairement aux rééditions sur papier des planches qui généralement comportent un texte contemporain de présentation, voir ci-dessus). On ne trouvera dans ce DVD que le texte brut et complet, les planches dans une définition haut de gamme, ainsi qu'un outil de recherche utile mais limité. On pourra regretter l'absence d'introduction substantielle ou de mise en perspective, mais on se consolera en espérant que cette édition suscitera de nouvelles études sur l'apport de cet ouvrage aux connaissances sur l'Égypte, mais aussi à I'histoire des sciences au XIX siècle.

16. L'ensemble des documents occupe un espace de 1,77 Go.

17. Voir en particulier http://gallica.bnf.fr/VoyagesEnAfrique/, sur la thématique des voyages en Afrique, où sont reproduits de nombreux auteurs, dont certains sont passés par l'Égypte. 
La réédition de la Description de l'Égypte dans un format plus qu'accessible (un DVD de 35 grammes), comparé aux deux seules éditions complètes du début du xix siècle, monumentales et fragiles, est donc une bonne nouvelle pour tous ceux qui s'intéressent, en historiens ou en amateurs, à l'Égypte depuis I'Antiquité jusqu'à la fin du xvIII siècle. Les planches d'histoire naturelle, dont certaines en couleur, par leur qualité, attireront autant les historiens des sciences que les passionnés. Cette édition, qui donne une nouvelle jeunesse à la Description de l'Égypte, devrait permettre de la débarrasser de ses bandelettes et de la sortir de son sarcophage.

Dernière minute : les textes et les planches de ce $D V D$, devant le succès qu'il rencontre, ont été mis en ligne sur le site de la Bibliotheca Alexandrina (http://descegy.bibalex.org/). Les mêmes outils de recherche dans le texte sont présents, mais les planches sont disponibles dans une résolution très inférieure à celle du DVD. 